\title{
UNA EXPLICACIÓN FORMAL DE LA ORIENTACIÓN Y DISTRIBUCIÓN DE LOS ADVERBIOS DE ACTOS DE HABLA*
}

\author{
SANG YOON KIM \\ Universidad Complutense de Madrid
}

\section{RESUMEN}

Este trabajo propone un análisis formal de los adverbios de actos de habla con consecuencias significativas para la comprensión de la interficie sintaxis-discurso. Los adverbios de actos de habla especifican el papel de los participantes del discurso en la enunciación, esto es, se orientan hacia el hablante o hacia el oyente. Argumentamos que estos adverbios se someten a estrictas restricciones formales como objetos localizados en la interficie sintaxis-discurso, y la orientación, considerada convencionalmente de índole pragmática, se determina mediante operaciones sintácticas idénticas a las que se observan en la Sintaxis Estricta. Presentamos un estudio pormenorizado de la orientación en diferentes tipos de oraciones a partir de una configuración más refinada de la periferia izquierda. Finalmente ofrecemos una propuesta sobre la ilegitimidad de los adverbios de actos de habla en las cláusulas subordinadas con el fin de evaluar la adecuación explicativa del presente análisis.

Palabras clave: adverbios de actos de habla, interficie sintaxis-discurso, periferia izquierda.

\section{ABSTRACT}

This paper proposes a formal analysis of speech act adverbs with relevant consequences for the understanding of the syntax-discourse interface. Speech act adverbs specify the role of discourse participants in the utterance process, that is, they are speaker- or hearer-oriented. It is argued that they are subject to strict formal restrictions as syntax-discourse interface items, and the orientation, conventionally regarded as a pragmatic phenomenon, is actually determined by syntactic opera-

\footnotetext{
* Deseo expresar mi profundo agradecimiento al Dr. D. Luis Sáez por el consejo y ánimo que me ha brindado en la realización de este trabajo. Asimismo agradezco enormemente las observaciones y sugerencias de mejora hechas por los revisores anónimos de la RSEL. Estoy también en deuda con Nuur Hamad por la corrección del texto en español. Cualquier tipo de error es solo atribuible al autor. Los primeros resultados del estudio fueron presentados en el XXXIX Simposio Internacional de la Sociedad Española de Lingüística en la Universidad de Santiago de Compostela.
} 
tions identical to the ones in Core Syntax. An account for the orientation in different types of sentences is developed under the proposal of a finer structure for the left periphery. Finally a specific proposal on the ungrammaticality of speech act adverbs in subordinate clauses is provided as a test of the explicatory adequacy of the general approach.

Key Words: left periphery, speech act adverbs, syntax-discourse interface.

\section{INTRODUCCIÓN}

Este trabajo propone un análisis formal de los adverbios de actos de habla (AAHs) con una periferia izquierda extendida que incorpora una configuración detallada para la interficie sintaxis-discurso ${ }^{1}$. Los AAHs, caracterizados por su interpretación orientada hacia el hablante Francamente, María canta muy bien o hacia el oyente Sinceramente, ¿canta bien Maria?, especifican la enunciación en una relación inseparable con los participantes del discurso, pero muestran al mismo tiempo que están sometidos a rígidas restricciones respecto a las condiciones relacionales y distribucionales, como se observará más adelante. Este comportamiento nos hace pensar que son objetos que se encuentran en la interficie sintaxis-discurso y que se requiere un enfoque gramatical sobre ellos. En este trabajo argumentamos a favor de que la orientación de los AAHs no es un fenómeno primitivo, sino que se decide en la sintaxis. Para ello, se asumen tres hipótesis principales:

(i) la periferia izquierda oracional se identifica como interficie sintaxis-discurso en la que el Sintagma Fuerza (SFu) linda con el dominio superior donde se organiza el discurso y el Sintagma Finitud (SFin) se pone en contacto con el inferior donde se representa el contenido oracional (Rizzi 1997);

(ii) los adverbios son especificadores de las proyecciones funcionales, cuya interpretación se determina por la información sintáctica de estas (Cinque 1999, 2004);

(iii) algunos rasgos de carácter pragmático son relevantes en la sintaxis (Speas y Tenny 2003, Hill 2007).

\footnotetext{
${ }^{1}$ Los adverbios de los que nos ocupamos se han presentado en la bibliografía con distintas denominaciones: adverbios orientados hacia el hablante (Jackendoff 1972, Dik, Hengeveld, Vester y Vet 1990, Ernst 2002), adverbios pragmáticos (Bellert 1977), adverbios orientados hacia el emisor o el receptor (Kovacci 1999, Torner Castell 2005), adverbios de actos de habla (Cinque 1999), adverbios de la manera del decir (Rodríguez Ramalle 2003), etcétera.
} 


\subsection{Información sobre los adverbios de actos de habla}

En español los adverbios francamente, honestamente, honradamente, sinceramente pueden ser interpretados como AAHs. Al orientarse hacia el hablante, los AAHs ponen de relieve que este se compromete a cumplir la cualidad denotada por su base adjetiva a la hora de proferir el enunciado. Si se orientan hacia el oyente, el hablante exige a su interlocutor que cumpla la misma condición al responder ${ }^{2}$. Los AAHs difieren en este punto de otros tipos de adverbios del ámbito oracional como los evaluativos o evidenciales. En el presente estudio delimitamos estrictamente el empleo del término «orientación» en el sentido que acabamos de describir ${ }^{3}$.

\subsection{Objetos del estudio: orientación y distribución}

Trataremos de dar cuenta de varios comportamientos de los AAHs en términos gramaticales. Algunos de ellos suelen definirse "por defecto" y otros apenas han recibido una atención particular en la bibliografía. Para ello, primero se formula una cuestión importante acerca del hecho de que solo es posible encontrar dos objetos de la orientación, esto es, el hablante y oyente, cuando podrían imaginarse varios candidatos potenciales para tal fenómeno como Dios, un oyente indirecto, la fuente de una noticia, etcétera.

Por otra parte, se suele afirmar que los AAHs se orientan hacia el hablante en las oraciones declarativas y hacia el oyente en las interrogativas. Sin embargo, una observación detenida indica que esto no es así. La orientación en las interrogativas no es unívoca. En otras palabras, puede dirigirse tanto al hablante como al oyente. (1b) y (1c) son dos paráfrasis posibles para $(1 \mathrm{a})^{4}$ :

(1) a. Francamente, ¿quieres casarte con María?

b. Sé franco al contestarme si quieres casarte con María.

c. Soy franco al preguntarte si quieres casarte con María.

\footnotetext{
${ }^{2}$ Como nos indica un revisor anónimo de la RSEL, también hay adverbios que se orientan a la forma o la manera de decir mediante su uso metalingüístico y que pueden considerarse como otro tipo de AAHs, p. ej., brevemente, sintéticamente, literalmente, exactamente, etc. Estos adverbios no forman parte del objetivo del presente estudio.

${ }^{3}$ Bellert 1977 señala asimismo que Jackendoff 1972 llegó a considerar que todos los tipos de adverbios oracionales, incluso los frecuentativos, se orientan hacia los papeles discursivos. Véanse Rodríguez Ramalle 2003 y Torner Castells 2005 para los criterios generales para la subclasificación de los adverbios oracionales y el inventario en español.

${ }^{4}$ Es importante notar que, al permitir dos paráfrasis como (1b) y (1c), (1a) no es más que una idéntica representación gráfica de dos construcciones diferentes, que se reflejan en la realización fonológica. Esta cuestión se analiza pormenorizadamente en el apartado 4.
} 
Otra pregunta capital que no se puede perder de vista surge respecto a la ilegitimidad de la presencia de los AAHs en las cláusulas subordinadas como se ilustra en (2). Nótese que no son capaces de establecer ninguna relación interpretativa con el hablante o el oyente, ni con el sujeto o el objeto indirecto de la cláusula matriz, a diferencia de lo que ocurre con los adverbios evaluativos o evidenciales en (3), que fijan dicha relación con el sujeto o el objeto indirecto de la cláusula matriz ${ }^{5}$ :

(2) a. *Juan afirma que, francamente, le da miedo la oscuridad.

b. *Juan pregunta a Ana si, sinceramente, le da miedo la oscuridad.

(3) a. Juan afirma que, desafortunadamente, le da miedo la oscuridad.

b. Juan pregunta a Ana si, ciertamente, le da miedo la oscuridad.

El trabajo está organizado del siguiente modo. En el apartado 2 se presenta una breve revisión crítica de estudios sobre los AAHs y el esquema sobre una nueva periferia izquierda del que se sigue la argumentación posterior. El apartado 3 analiza la orientación de los AAHs en torno a las oraciones declarativas e interrogativas. Se observa que la orientación se determina mediante complejas computaciones sintácticas, al mismo tiempo que se descarta el supuesto habitual de que los AAHs son modificadores del verbo de dicción implícito. En el apartado 4 se muestra que la intervención de la sintaxis es mucho más pertinente en la organización de diferentes tipos de oraciones interrogativas y que a ello se debe la orientación de los AAHs hacia el hablante en ciertas oraciones interrogativas. Se estudia en el apartado 5 la ilegitimidad de los AAHs en las cláusulas subordinadas a partir de la distinción de las periferias izquierdas en función de la codificación de la fuerza ilocutiva en este dominio. En el último apartado se presentan las conclusiones del estudio.

\footnotetext{
${ }^{5}$ Con el verbo decir en la cláusula matriz estas construcciones muestran una mayor aceptabilidad en cuanto a la orientación de los AAHs hacia el sujeto de la cláusula matriz -cf. (i)-. Se ha señalado en la bibliografía que este tipo de verbos no forma una auténtica subordinación sino parataxis, fenómeno que no vamos a tratar en este trabajo (cf. Torrego y Uriagereka 1992).

(i) ?El jefe dice que, sinceramente, el negocio se encuentra en plena crisis.

Como nos señala un revisor anónimo de la RSEL, una prueba de que la aceptación de los adverbios en este caso depende de la naturaleza cuasi-paratáctica de la construcción es que el dato empeora notablemente si hay marcas formales de una relación claramente hipotáctica, p. ej., una consecutio temporum, como se observa en el siguiente ejemplo:
}

(ii) ?*El jefe dijo que, sinceramente, el negocio se encontraba en plena crisis.

Véase la nota (26) para más información relacionada con esta cuestión. 


\section{CONSIDERACIONES PRELIMINARES}

\subsection{Los adverbios de actos de habla en el dominio periférico}

Cinque 1999 propone que los AAHs ocupan la posición jerárquicamente más alta en el dominio oracional. Se basa principalmente en que estos adverbios parecen seguir a los tópicos y focos. Es posible, señala, que los precedan mediante el desplazamiento hacia el especificador del SFu, si se separan del resto de la oración por una pausa (Cinque 1999, p. 84):

(4) a. Di questo, A NESSUNO francamente potrei parlare. 'De esto, A NADIE, francamente, podrías hablar'.

b. Francamente, di questo, A NESSUNO potrei parlare. 'Francamente, de esto, A NADIE, podrías hablar'.

Sin embargo, esta explicación resulta cuestionable por varias razones. Primero, desde el punto de vista sintáctico, la derivación de (4b) a partir de (4a) es incompatible con la hipótesis del propio autor de que los adverbios se ensamblan en la base y no pueden desplazarse por sí solos. Si bien se puede defender algún desplazamiento masivo que puede incluirlos, no se encuentra ningún motivo para tal movimiento en este contexto. Segundo, prosódicamente, se puede percibir la misma pausa tras francamente en ambos ejemplos. Sería difícil notarla con los adverbios modificadores del verbo, por lo que la coma de (4b) parece ser característica propia y general de los AAHs, y no una propiedad particular de (4b) resultante de un supuesto movimiento ${ }^{6}$. Nótese, además, que la secuencia de (4b) es la que se realiza con entonación neutra. Proponemos, a partir de estas observaciones, que son el tópico y el foco los que se han desplazado hacia la izquierda del adverbio, que se queda in situ, derivándose así (4a) a partir de (4b). Dado el orden de palabras en (4b), los AAHs ocupan una posición más prominente que el tópico y el foco en la periferia izquierda.

Rodríguez Ramalle 2003 propone también que los AAHs se generan en la periferia izquierda, precisamente, en el especificador del $\mathrm{SFu}$, tras observar que se encuentran al margen de las modalidades oracionales ${ }^{7}$.

${ }^{6}$ Kovacci 1999 habla de la inestabilidad del uso de la coma como señal de las unidades prosódicas separadas.

${ }^{7}$ Sin embargo, como se ilustra en (ii), los AAHs pueden seguir a la oración interrogativa siempre que estos queden, entonativa e interpretativamente, fuera de la interrogación:

(i) Francamente, ¿a qué hora ha pasado ese hombre por aquí?

(ii) ¿A qué hora ha pasado ese hombre por aquí, francamente?

La presencia de entonación marcada en (ii) sugiere la intervención de operaciones sintácticas adicionales. Si se asume que el movimiento sintáctico se permite solo hacia la izquier- 
No obstante, el análisis de los AAHs como especificador del SFu no puede dar cuenta de su inaceptabilidad en las cláusulas subordinadas -cf. (2)-, y daría lugar a una configuración anómala como la siguiente:

(5) Juan afirma $\left[\mathrm{SFu}\left({ }^{*}\right.\right.$ francamente) $\left[\mathrm{Fu}^{\circ}\right.$ que $[\mathrm{ST} \ldots$

Una secuencia con el adverbio así representado no puede tener ninguna materialización real. La oración (6) se ajusta a ese orden lineal, pero en ella no llega a interpretarse francamente como parte de la subordinada, y el adverbio tiene en su alcance toda la oración al haber sido generado originalmente en la periferia izquierda de la cláusula matriz:

(6) Juan afirma, francamente, que le da miedo la oscuridad.

Aunque los datos apuntan a que los AAHs se encuentran en el dominio periférico oracional, parece que un análisis basado en esta área no puede alcanzar una explicación satisfactoria, lo que consideramos que se debe a una concepción actual de la periferia izquierda excesivamente simple. Pasaremos, entonces, a revisar hipótesis alternativas donde se amplíe y refine aún más esta interficie sintaxis-discurso.

\subsection{La relevancia sintáctica de algunos constituyentes discursivos}

Speas y Tenny 2003 se centran en aquellas propiedades pragmáticas gramaticalmente relevantes y en la estructura sintáctica pragmáticamente relevante. Observan que la sintaxis impone restricciones sistemáticas a la organización de los elementos discursivos en la periferia izquierda, lo que no sería esperable, si estos estuvieran condicionados únicamente por la pragmática. A partir del paralelismo entre las interficies léxico-sintaxis y sintaxis-discurso, proponen que ciertos rasgos interpretativos se generan en el dominio periférico oracional como núcleos de dos proyecciones predicativas incidentes en el acto de habla, de manera similar a como los rasgos verbales proyectan S $v$ y SV. Se denominan Sintagma del Acto de Habla (SAH) y Sintagma del Acto de Habla* $\left(\mathrm{SAH}^{*}\right)$. En estas proyecciones papeles pragmáticos (papeles-p) como el hablante (papel-H), el oyente (papel-O) y un papel logofórico se interpretan estructuralmente de modo análogo a los papeles- $\theta$ en el dominio del $\mathrm{S} v$ y $\mathrm{SV}^{8}$.

da (Kayne 1994) junto con la imposibilidad del desplazamiento de los adverbios (Cinque 1999), se puede afirmar que (ii) es el resultado del movimiento de la interrogativa entera en torno al adverbio en (i).

${ }^{8}$ Sells 1987 señala que hay tres tipos de antecedentes logofóricos jerárquicamente ordenados, esto es, source (el participante que proporciona la información), self (el participan- 
Esta hipótesis ha sido examinada detenidamente en Hill 2007, quien reorganiza el dominio del SAH y argumenta que hay categorías funcionales que marcan a los papeles de hablante y oyente. El papel-H y el papel-O proyectan como núcleo su propia proyección: el Sintagma Papel de Hablante $(\mathrm{SPaH})$, que sirve para exclamativos de punto de vista o emoción como Dios mío, madre mía, y el de Oyente ( $\mathrm{SPaO})$, en que se ubican los vocativos. Se proyectan en el especificador del SAH y SAH*, respectivamente $^{9}$ :

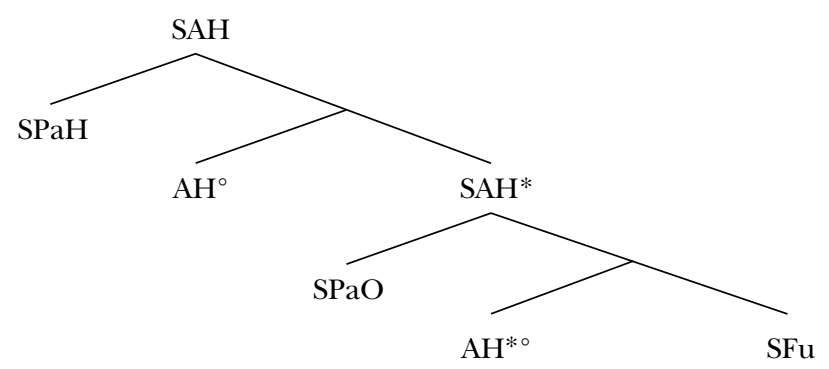

En las siguientes secciones llevaremos hasta sus últimas consecuencias estas consideraciones para dar cuenta del comportamiento de los AAHs. No obstante, diferimos de Hill al rechazar que el SAH* seleccione el SFu y al defender que este sigue siendo la proyección más prominente en la periferia izquierda. Volveremos sobre esta cuestión más adelante (cf. §5). De momento, nos limitamos a señalar que el SAH y

te del que se expresa el estado mental) y pivot (el participante del que está expresado el punto de vista físico en el evento). Speas y Tenny 2003 precisan que, al formar parte de los papeles relevantes en el discurso, solo puede ser elegido uno de ellos para sumarse al hablante y el oyente. Se refuerza así el paralelismo entre el dominio verbal y el interpretativo en que ambos no pueden tener más de tres papeles sintácticamente representados.

${ }^{9}$ Según la propuesta de la autora mencionada, cada núcleo Papel toma al SD como complemento para borrar su rasgo no interpretable. Sin embargo, queda por averiguar la identidad de este complemento. Parece que los vocativos no proyectan SD (cf. Longobardi 1994). Aunque en algunas lenguas escandinavas los vocativos aparecen con un determinante afijado, como se ve en (i), se considera que este determinante ocupa el núcleo de una proyección diferente del SD por debajo de las proyecciones de adjetivos en las construcciones nominales (Roehrs 2006, p. 74):

$\begin{array}{ll}\text { (i) dumme idiot-en } & \text { (bokmål) } \\ & \text { estúpido idiota-DET }\end{array}$

Además, nos parece que el SN en los vocativos es defectivo, si asumimos que los adjetivos ocupan las proyecciones extendidas de N (cf. Longobardi 1994). Los vocativos no llevan modificadores a excepción de algunos casos restringidos en la lengua escrita como los encabezamientos de cartas (querido amigo mío, estimados señores clientes). El uso de los adjetivos como vocativos está limitado también a un número exiguo de lexemas convencionales (;Hola, guapa!/\#;Hola, sublime!; ¿Qué tal, majo?/\#_Qué tal, estupendo?). 
$\mathrm{SAH}^{*}$ preceden al STop y SFoc, dada la distribución de los constituyentes correspondientes a estas proyecciones:

(8) a. Dios mío (excl.), Juan (voc.), ese premio, A ANA se lo han dado.

b. Madre del amor hermoso (excl.), niños (voc.), ¿se puede saber qué está pasando aquî́?

\section{LA SINTAXIS EN LA INTERPRETACIÓN DE LOS ADVERBIOS DE ACTOS DE HABLA}

\subsection{La relación de sondeo en la orientación}

El fenómeno de que los AAHs solo se orientan hacia dos papeles-p sugiere la posibilidad de que estos adverbios y papeles estén implicados en una relación exclusiva de selección. Recuérdese que Speas y Tenny 2003 señalan que son tres los papeles-p gramaticalmente representados: el hablante, el oyente y un papel logofórico (cf. §2.2). Dado que los papeles logofóricos reservan su propia función para la evidencialidad (Sells 1987, Speas 2004), se explica a priori la razón por la que los AAHs no pueden tener más de dos objetos de la orientación y estos deben ser hablante y oyente ${ }^{10}$.

Ahora bien, proponemos que los AAHs se ensamblan en la posición de especificador de la proyección funcional SX situado entre SFu y SAH, cuyo núcleo X tiene un rasgo no interpretable [papel], crucial para identificar al individuo relevante en el acto de habla. Este rasgo sondea un papel concreto de naturaleza discursiva que le pueda asignar el valor y selecciona entre hablante y oyente, que son los dos únicos papeles-p válidos para dicho objetivo ${ }^{11}$. Es de esperar, entonces, que la interpreta-

\footnotetext{
${ }^{10}$ El hablante y el oyente son nociones en el universo de discurso, mientras que el papel-H y el papel-O son rasgos sintácticos. Sin embargo, utilizamos indistintamente los términos de las dos clases, siempre que esto no perjudique nuestro argumento.

${ }^{11}$ Los autores minimistas vienen asumiendo que las lenguas naturales, como imperfección inherente, exhiben ciertos rasgos formales no interpretables, esto es, rasgos sin contenido semántico, que deben ser eliminados antes de que las derivaciones sintácticas accedan a los niveles de interficie entre la facultad de lenguaje y el sistema de actuación intencional-conceptual. En una versión anterior del Programa Minimista (Chomsky 1995) se hacía uso de la noción de «cotejo» para explicar la computación sintáctica por la que se borraban dichos rasgos. Simplificando mucho, el cotejo es un proceso por el cual un rasgo no interpretable de una categoría-núcleo determinada atrae hasta su posición de especificador a otra categoría suficientemente próxima y con un rasgo equivalente pero interpretable, de modo que el segundo pueda asignar valor al primero $y$, como consecuencia, borrarlo a través de una relación estructural núcleo-especificador. Al cotejo lo sustituyó posteriormente la relación de «sondeo» (Chomsky 2000): ahora, el rasgo no interpretable, en calidad de sonda, busca una meta dentro del dominio de la categoría a la que pertenece. La meta es la categoría más cer-
} 
ción de los AAHs, que son manifestaciones léxicas de esta proyección funcional (cf. Cinque 1999), se decida en función del papel-p con el que se establezca la relación de sondeo mediante la configuración núcleoespecificador. Las proyecciones se representan del modo siguiente:

(9)

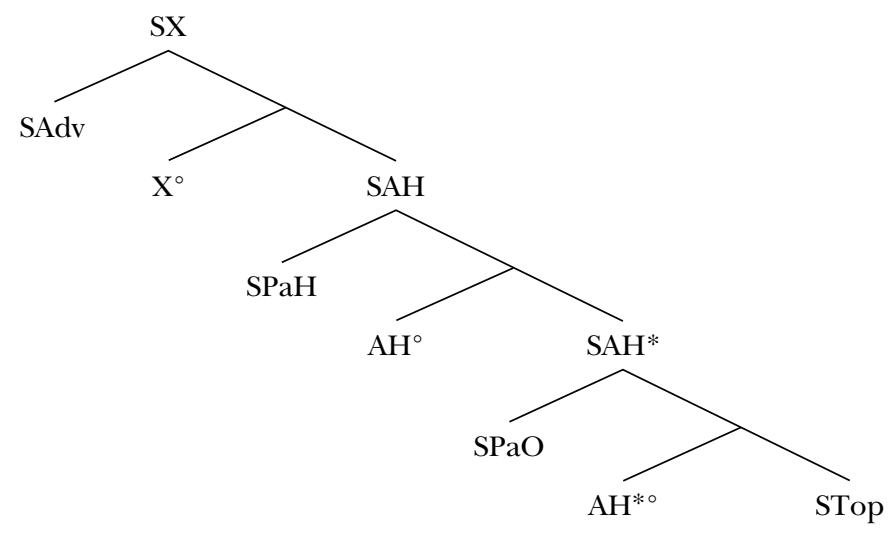

Esta configuración presenta cierta ventaja extra al ajustarse a ciertas propiedades de los AAHs observadas en secciones anteriores, como su alcance oracional o su independencia con respecto a las modalidades oracionales (interrogación, exclamación, declaración, etc.). Nótese, además, que ahora el paralelismo entre el dominio periférico del SAH y el oracional del $\mathrm{S} v$ se extiende hacia las categorías superiores de ambos dominios. Es decir, X sondea un constituyente con papel-p a fin de borrar el rasgo no interpretable, de manera idéntica a como T sondea un constituyente con papel- $\theta$ con el mismo objetivo.

Los AAHs se orientan unívocamente hacia el hablante en las oraciones declarativas. Este fenómeno se debe a que el rasgo [papel] de $\mathrm{X}$ sondea al papel-H en el especificador del SAH, que es el objeto más cercano con el rasgo equivalente en su dominio. De este modo el hablante se califica de papel relevante y el AAH se interpreta, consecuentemente, orientado hacia él. La flecha en (10b) indica el sondeo que se efectúa en la derivación de una oración como (10a):

\footnotetext{
cana a la sonda con un rasgo correspondiente interpretable que le pueda asignar valor tras ser identificado por ella. La reinterpretación del cotejo en términos de sondeo aporta sencillez y uniformidad en términos teóricos, puesto que la eliminación del rasgo no interpretable se determina únicamente por los propios rasgos implicados sin el recurso adicional del movimiento, complejidad computacionalmente necesaria en el modelo anterior. Recurrimos a este nuevo método para fundamentar nuestra argumentación en estas páginas. Para un punto de vista diferente que aboga por el vigor de la perspectiva anterior basada en el movimiento, véase Hornstein 2009, cap. 6; para una perspectiva basada en el sondeo distinta a la aquí asumida, véase Pesetsky y Torrego 2007.
} 
(10) a. Francamente, Juan escribió esa novela.

b. $\left[\mathrm{SX}[\mathrm{SAdv}]\left[\mathrm{X}^{\prime}\left[\mathrm{X}^{\circ}\right]\left[\mathrm{SAH}\left[\mathrm{SPaH}\left[\mathrm{PaH}^{\circ}\right]\right]\left[\mathrm{AH}^{\prime}\left[\mathrm{AH}^{\circ}\right] \ldots[\mathrm{ST} \ldots\right.\right.\right.\right.$

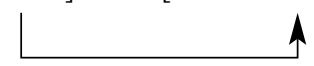

En cuanto a la orientación hacia el oyente en las interrogativas, es necesario precisar el estado del papel-H en este tipo de oraciones. Proponemos que este papel permanece implícito en las interrogativas. Nuestra idea se sigue de que la computación en el dominio de X para formar las interrogativas se puede equiparar a aquella en el dominio de $\mathrm{T}$ para construir la voz pasiva. La referencia a esta semejanza ya tiene antecedente en Speas y Tenny 2003, quienes consideran que el tema o el destinatario son los papeles- $\theta$ relevantes en la pasiva, y el oyente, que es el destinatario en el acto de habla, es el papel-p relevante en las interrogativas. Las autoras intentan explicar la analogía adoptando el modelo de Larson 1988 sobre la construcción de doble objeto: el papel-O, complemento de $\mathrm{AH}^{*}$ en su modelo, pasa por una operación similar a la alternancia dativa y se mueve al especificador de SAH*, rigiendo así el contenido del enunciado degradado y, por tanto, desencadenando la orientación hacia sí mismo. Sin embargo, su uso de operaciones como la adjunción a la derecha, ya presente en Larson, o el recurso a los múltiples especificadores, son aspectos que se apartan de los cánones más generalmente aceptados de la gramática actual (cf. Kayne 1994).

Nosotros, siguiendo la versión revisada en torno a la configuración del SAH y del SAH*, nos proponemos dar cuenta de la mencionada analogía con la pasiva a través de estrategias deductivas. Nos servimos del análisis de Kalluli 2007, en el que se señala que el agente queda implícito en la pasiva porque el rasgo [-argumento explícito] del núcleo $v$ impide que el SD explícito se ensamble en el especificador del Sv. Semejantes reflexiones se encuentran en Alexiadou, Anagnostopoulou y Schäfer 2006, quienes descomponen los verbos de cambio de estado y proponen que hay un componente, llamado Voz, responsable de introducir el argumento externo. Si el núcleo Voz es activo, los papeles como agente o causa se realizan en su especificador, mientras que, si es pasivo, son implícitos. Si es correcto el paralelismo entre los dominios de X y T, defendido a lo largo de este trabajo, el análisis sobre el agente implícito en la voz pasiva se podría aplicar mutatis mutandis al papel-H en las interrogativas.

Proponemos en concreto que la implicitud del papel-H hace que el sondeo del rasgo no interpretable de $\mathrm{X}$ pase por alto el estrato de $\mathrm{SAH}$ y busque como meta propia el próximo objeto que sea compatible, esto es, el papel-O en el especificador de SAH*. Como consecuencia los AAHs presentan orientación hacia el oyente en las interrogativas. El papel-H implícito se indica entre paréntesis en la siguiente representación: 
(11) a. Francamente, ¿escribió Juan esa novela?

b. $\left[\mathrm{SX}[\mathrm{SAdv}]\left[\mathrm{x}^{\prime}\left[\mathrm{X}^{\circ}{ }_{[\mathrm{SAH}}\right]\left[\mathrm{SAH}\left[\mathrm{SPaH}\left[\left(\mathrm{PaH}^{\circ}\right)\right]\right]\left[\mathrm{SAH}^{*}\left[\mathrm{SPaO}\left[\mathrm{PaO}^{\circ}\right]\right]\right.\right.\right.\right.$ $[\mathrm{SFOC} \ldots$

Se demuestra de este modo que la orientación de los AAHs no es un fenómeno que tenga lugar por defecto, ni se caracteriza únicamente por ser discursiva, sino que deriva de condiciones impuestas por la sintaxis ${ }^{12}$.

\subsection{Más allá del $S X$}

Nuestra propuesta predice que los AAHs precederán a los exclamativos y vocativos, puesto que el SX selecciona al SAH y SAH*, cuyos especificadores constituyen las posiciones en las que se ubican estas expresiones. Sin embargo, esta predicción no parece en principio confirmada por los datos, como revelan los ejemplos siguientes ${ }^{13}$ :

(12) a. (Dios mío/Juan,) sinceramente, (*Dios mío/Juan,) no lo entiendo.

b. (Madre mía/tío,) francamente, (*Madre mía/tío,) esto es un lío.

c. (Jo/oye,) honestamente, (*jo/oye,) esta novela es mediocre.

La distribución en la que los exclamativos y vocativos preceden a los AAHs se explica mediante el desplazamiento de estos constituyentes a una posición todavía más alta que la del SX, que etiquetamos SF, por

\footnotetext{
12 Cabría pensar en una alternativa basada en los rasgos componentes de persona, que se especificarían del siguiente modo según las personas gramaticales (Halle 1997, p. 129):

(i)

\begin{tabular}{|l|c|c|c|}
\cline { 2 - 4 } \multicolumn{1}{c|}{} & 1 & 2 & 3 \\
\hline Participante del acto de habla & + & + & - \\
\hline Autor del acto de habla & + & - & - \\
\hline
\end{tabular}

De asumir que los rasgos relevantes de primera persona se extienden al hablante y los de segunda al oyente, y que $\mathrm{X}^{\circ}$ sondea al rasgo [+participante], se obtendría el mismo tipo de explicación que la expuesta en el texto. Sin embargo, la identificación de estos rasgos de las personas gramaticales y los de los papeles-p es algo dudosa, sobre todo porque al coincidir el hablante y el sujeto oracional (p. ej., yo), habría dos categorías redundantes con los mismos rasgos. Parece, en efecto, que la diferenciación entre hablante/oyente, por un lado, y prime$\mathrm{ra}$ /segunda persona, por otro, es una cuestión excesivamente intrincada no solo en el ámbito formal, sino también en otras vertientes de investigación lingüística. Dejamos esta cuestión para una investigación posterior.

${ }^{13}$ En este apartado solo hablaremos de las oraciones declarativas. Las expresiones exclamativas en las oraciones interrogativas comportan ciertas computaciones más complejas a las que volveremos en el siguiente apartado.
} 
algún rasgo de carácter enfático claramente evidenciado en el acento de foco que ostentan. Hacemos notar, de paso, que la anteposición es obligatoria para los exclamativos y opcional para los vocativos ${ }^{14}$.

Como hemos visto con el SF, es probable que existan más categorías funcionales por encima del SX, según sugieren los ejemplos siguientes además del ejemplo (ii) de la nota 7 :

(13) a. Me pone mal este tipo de películas, francamente.

b. Mi hermana, sinceramente, salió en defensa del tonto de su marido.

Recuérdese que los adverbios no pueden desplazarse por sí solos (Cinque 1999). La explicación razonable sobre el orden de palabras en (13) es que las secuencias que preceden a los AAHs han abandonado su posición de origen, que se encuentra linealmente a la derecha de los adverbios. El ejemplo (4a) también se puede entender en este contexto ${ }^{15}$. Sin pretender ser exhaustivos en la explotación de esta zona adicional, dejamos abierta su organización minuciosa, que cae fuera del alcance del presente trabajo.

\subsection{Los adverbios de actos de habla no son modificadores del verbo de dicción implícito}

No han sido pocos los autores que han mantenido que los AAHs son modificadores de un verbo de dicción implícito con valor ilocutivo (Hengeveld 1990, Kovacci 1991, 1999, Báez San José 1994, Kaul de Marlangeon 2002, Torner Castells 2005). Partiendo de la idea de que (14a) derivaría de (14b) sin cambio alguno en la estructura, AAHs como el de (14c) se equipararían con adverbios de modo verbal de un verbo de dicción explícito, como se manifiesta en (14d):
(14) a. No quiero comer aquí.
b. Digo que no quiero comer aquí.
c. Francamente, no quiero comer aquí.
d. Digo francamente que no quiero comer aquí.

Sin embargo, diversos estudios de gramática han señalado en varias ocasiones los inconvenientes de suponer un verbo de dicción implícito ${ }^{16}$.

\footnotetext{
${ }^{14}$ Sobre la causa de esta diferencia no tenemos explicación en este momento. Una explicación de la anteposición de los vocativos a partir de un movimiento fonológico prediría la ausencia de efectos interpretativos.

${ }_{15}$ Véase Hill 2007, p. 2103, para una categoría funcional de carácter tópico superior al SAH.

${ }_{16}$ Para las críticas generales sobre el verbo de dicción implícito propuesto en el marco de la semántica generativa (Ross 1970, Sadock 1974), remitimos al lector a Anderson 1971 y Fraser 1974.
} 
Aparte de la dificultad de determinar cuál es el verbo adecuado, una de las graves consecuencias de identificar las oraciones en (14a) y (14b) sería el carácter circular de la aparición de dicho verbo (cf. Speas y Tenny 2003). Si estas fueran idénticas, nada impediría decir que (14b) se identificaría a su vez con (15a). Por tanto, sería obligado admitir que digo podría multiplicarse sin límite -cf. (15b) - solo para tener al final el mismo significado que (14a):

(15) a. Digo que digo que no quiero comer aquí.

b. Digo que digo que [...] digo que no quiero comer aquí.

En concreto, surge un problema conceptual respecto a la orientación de los AAHs cuando pasamos a preguntarnos cómo se puede explicar la orientación hacia el oyente en las oraciones interrogativas. La orientación, según la postura en cuestión, debería dirigirse al hablante incluso en este tipo de oraciones, puesto que es él quien profiere la pregunta y, por tanto, el sujeto del verbo de dicción implícito del que un AAH sería modificador.

Para dar cuenta de esta paradoja se ha sugerido, p. ej., en Torner Castells 2005, que hay dos actos ilocutivos diferentes en esta ocasión, pero ligados entre sí, y que los AAHs se orientan catafóricamente hacia el hablante del segundo, esto es, el oyente del primero. Pero nos parece que la interacción de dos planos de actos de habla distintos y la alternancia de la relevancia de papeles a partir de la suposición anticipada del acto perlocutivo se alejan notoriamente de una explicación de corte formal, que es lo que pretende obtener el presente trabajo. Por consiguiente, se descarta en estas líneas la idea de que los AAHs sean modificadores modales del verbo ilocutivo que se encuentra implícito. Hemos presentado, en cambio, una alternativa que se centra en los rasgos formales inherentes, los cuales determinan el comportamiento de los AAHs ${ }^{17}$.

17 Un revisor de la RSEL hace la interesante observación de que los AAHs no admiten alternativa con prefijación negativa -cf. (ii)-. La restricción no parece simplemente de índole morfológica, ya que el empleo de la forma negativa como adverbio de manera verbal es legítimo -cf. (i)-:

(i) Juan se comportó deshonestamente.

(ii) a. *Deshonestamente, me gustó el discurso del alcalde en la ceremonia.

b. *Insinceramente, ¿por qué te desagrada tanto mi amigo?

De momento no tenemos una respuesta para esta observación, que dejamos para futuras investigaciones. Sin embargo, creemos que esta restricción podría reforzar nuestro argumento de que los AAHs no son modificadores de un verbo de dicción implícito, dado que tales adverbios con prefijación negativa pueden figurar como modificadores de los verbos de dicción:

(iii) ?Juan \{dijo/manifestó/afirmó\} \{deshonestamente/insinceramente\} que María fue la que robó las gallinas. 


\section{ORACIONES INTERROGATIVAS ATRIBUIDAS Y ADVERBIOS DE ACTOS} DE HABLA

\subsection{La implicación sintáctica del hablante en las oraciones interrogativas}

No sería de esperar, según la propuesta presentada en las secciones anteriores, que los AAHs se orientaran hacia el hablante en las oraciones interrogativas, ya que el estado implícito del papel-H impediría este fenómeno lingüístico. Sin embargo, al comentar que la orientación de los AAHs en las interrogativas puede variar, se ha señalado que el hablante es un posible objeto de la orientación también en este tipo de oraciones, tal como se muestra en las paráfrasis (16c) y (17c) (véase también la nota 4):

(16) a. Francamente, ¿quieres casarte con María?

b. Sé franco al contestarme si quieres casarte con María.

c. Soy franco al preguntarte si quieres casarte con María.

(17) a. Sinceramente, ¿quién no sabe de eso?

b. Dime sinceramente quién no sabe de eso.

c. Te pregunto sinceramente quién no sabe de eso.

Se trata de las interrogativas «atribuidas», que se distinguen de las interrogativas que tratamos en el apartado anterior y que llamaremos «regulares». En las interrogativas regulares el hablante trata de conseguir informaciones que necesita a través de su interlocutor. En cambio, en las atribuidas el hablante no busca información de manera inocente como en el otro caso, sino que quiere verificar la información que ya tiene a su alcance sobre el asunto ${ }^{18}$. La orientación de los AAHs se dirige al hablante en este último caso (cf. Kovacci 1999). Se podrían describir algunas situaciones correspondientes a los ejemplos, teniendo en cuenta que la interrogación no es siempre sinónimo de pregunta: en (16b) el hablante manifiesta nítidamente su curiosidad o interés acerca de la disposición de su interlocutor a contraer matrimonio con María y en (16c) el hablante hace la interrogación sobre la misma disposición, pero ya la da por sentada; en (17b) el hablante se siente intrigado de manera transparente por saber si hay alguien que no sepa del asunto y en (17c) expresa su creencia de que no hay nadie que no se haya enterado del asunto.

A primera vista, la distinción entre las interrogativas regulares y las atribuidas parece ser de índole pragmática. Sin embargo, argumenta-

\footnotetext{
${ }^{18}$ Escandell Vidal 1999, p. 3978, da la siguiente definición para las interrogativas atribuidas: «[...] aquellas interrogativas por medio de las cuales el emisor hace oír palabras efectivas o supuestamente pronunciadas por otro hablante».
} 
mos en este apartado a favor de que la interficie sintaxis-discurso se organiza de diferente manera en cada caso, es decir, la diferencia entre interrogativas atribuidas y regulares se corresponde con diferencias de orden computacional que la motivan. El hecho de que una interrogativa atribuida se enuncie como manifestación de júbilo, sorpresa, reproche, molestia, etc., del hablante dependerá del contexto discursivo, pero hay más sintaxis de lo que parece en la propia organización de esta clase de interrogativas, que se distinguen estructuralmente de las regulares, como observaremos en seguida. Concretamente, proponemos que las interrogativas atribuidas, estructuralmente más complejas, mantienen intacto el papel-H y este es el elemento que singulariza de manera decisiva a estas interrogativas en términos gramaticales.

El comportamiento de los AAHs en las interrogativas atribuidas debe ser entendido en este contexto como reflejo de la mencionada diferencia estructural. Es conveniente, primero, fijarnos en que los AAHs se presentan con diferentes características prosódicas. En las interrogativas regulares la pausa que divide el adverbio y el resto de la oración es menor y la entonación del adverbio se integra en cierta medida en el contorno entonativo de la oración entera, mientras que la pausa en las atribuidas es notablemente mayor y la entonación del adverbio presenta cierto grado de descenso. Por otra parte, dos adverbios que pertenecen a los AAHs pueden coaparecer en una misma interrogativa atribuida con diferentes orientaciones $-(\mathrm{H})$ indica la orientación hacia el hablante y $(\mathrm{O})$ hacia el oyente-:

\section{(18) Francamente $(\mathrm{H})$, sinceramente $(\mathrm{O})$, ¿quieres casarte con María?}

Parece claro, entonces, que tanto la diferencia prosódica como la posibilidad de coaparición indican que el adverbio orientado hacia el hablante no compite con el orientado hacia el oyente por la misma posición en estas oraciones. Efectivamente, hay algo más que justifica la presencia del papel-H en las interrogativas atribuidas. Consideremos ahora los casos en los que la oración interrogativa aparece acompañada por el exclamativo, que es otra materialización del SPaH:

(19) a. Madre del amor hermoso, ¿por qué no dejas de decir disparates?

b. Por Dios, ¿a quién le va a importar este asunto?

c. Anda, ¿te vas a Alemania?

Cualquiera de estas interrogativas privadas del exclamativo puede ser interrogativa regular o atribuida en función del contexto en que se enuncie, y una vez marcada con la entonación que le corresponda. En cambio, la presencia del exclamativo las caracteriza sistemáticamente como atribuidas. Nótese que ninguno de los ejemplos puede pro- 
ferirse con el fin de obtener del interlocutor una información desconocida $^{19}$. Esta observación se traduce en la idea de que gran parte de la interpretación básica de las interrogativas queda legitimada mediante pautas sintácticas, es decir, a partir de la representación presente en la interficie sintaxis-discurso del papel-H.

\subsection{La explicación formal del fenómeno}

En lo que respecta a las interrogativas atribuidas y los AAHs en estas oraciones proponemos lo siguiente:

(i) el dominio sintáctico computacionalmente generado donde se legitima el papel-H llega a tener alcance sobre otro dominio que se correspondería con una oración interrogativa regular;

(ii) el adverbio orientado hacia el hablante en estas oraciones se genera en la base en el primer dominio.

Este planteamiento puede ser implementado técnicamente mediante el concepto de «fase» (Chomsky 2000, 2001). Dado que la periferia izquierda que hemos ido extendiendo en este trabajo es la forma refinada del SC, el criterio de fase puede ser aplicado a las construcciones encabezadas por el SFu. Es decir, la interrogativa regular es una fase completa (SFu2) en la que solo el papel-O es accesible a la computación sintáctica. Dicho papel dejará de ser accesible a la computación una vez el complemento del núcleo de fase Fu2, que lo incluía, quede clausurado tras ensamblarse en la estructura el núcleo de la fase ulterior, Fu1 (cf. Nissenbaum 2000). En este nuevo estadio de desarrollo estructural emergerá el papel-H explícito, y el SFu1 se interpretará como interrogativa atribuida. Como ilustración, representamos a continuación la configuración de la oración interrogativa atribuida de (18), donde dos AAHs con diferentes orientaciones están realizados como manifestación léxica de las proyecciones incidentes:

(20) $\left[\mathrm{SFu} 1\left[\mathrm{SF} 1\left[\mathrm{SX} 1\right.\right.\right.$ francamente $\left[\mathrm{SAH} 1[\mathrm{SPaH} 1]\left[\mathrm{AH} 1^{\prime}\left[\mathrm{AH}^{\circ}\right]\left[\mathrm{SAH}^{*} 1\right.\right.\right.$ $\left[\mathrm{SFu} 2\left[\mathrm{SF} 2\left[\mathrm{SX} 2\right.\right.\right.$ sinceramente $\left[\mathrm{SAH} 2[(\mathrm{SPaH} 2)]\left[\mathrm{AH} 2{ }^{\prime}\left[\mathrm{AH} 2^{\circ}\right]\left[\mathrm{SAH}^{*} 2\right.\right.\right.$ [SFoc quieres...

Como hemos dicho, la fase encabezada por el SFu2 aporta el contenido semántico y fonológico de la interrogación regular tomando

\footnotetext{
${ }^{19}$ Los ejemplos de (19) tenderían a rechazar, al contrario del ejemplo (18), la presencia de AAHs orientados al oyente, lo que corroboraría nuestro análisis de que estas interrogativas son indiferentes al hecho de realizarse para obtener información. Por ahora no tenemos respuesta a por qué las interrogativas atribuidas con exclamativo desfavorecen la presencia de los adverbios orientados al oyente. Le agradecemos a un revisor anónimo de la RSEL el habernos llamado la atención sobre esta cuestión.
} 
como base la secuencia creada en el dominio de su SFoc. La fase superior convierte toda la oración en una interrogativa atribuida mediante la relevancia adquirida por el papel del hablante. Es en esta fase donde se legitiman tanto el AAH orientado hacia el hablante como el exclamativo $^{20}$.

Este análisis aporta otras ventajas explicativas. La primera tiene que ver con el orden de palabras entre los AAHs y el vocativo. Considérese el caso en el que el vocativo generado dentro del SFu2 se ha desplazado al inicio de la oración. Ello no viola la Condición de Impenetrabilidad de la Fase (Chomsky 2001), ya que se trata de un movimiento cíclico que se produce a través del especificador de SFu2, como sucede con la extracción del elemento-Cu desde una cláusula subordinada. El vocativo se moverá finalmente desde el especificador del SFu2 al especificador de SF1.

(21) Juan, francamente $(\mathrm{H})$, sinceramente $(\mathrm{O})$, ¿quieres casarte con María?

Además, se ofrece así una explicación coherente para el hecho de que las oraciones declarativas no acepten dos AAHs, cada uno orientado hacia un papel-p diferente. Para formar tal oración debería satisfacerse el requisito de que estuviera disponible una fase correspondiente a una declarativa con AAH orientado hacia el oyente, y posteriormente subsumida en otra fase superior. Ya se ha señalado, sin embargo, que en las declarativas la orientación de los AAHs se dirige necesariamente al hablante, cuyo valor resulta activo para la relación de sondeo.

\section{LA INACEPTABILIDAD DE LOS ADVERBIOS DE ACTOS DE HABLA EN LAS CLÁUSULAS SUBORDINADAS}

\subsection{Consideraciones preliminares}

Hemos mencionado previamente algunos datos que ilustraban la inaceptabilidad de la presencia de los AAHs en las cláusulas subordina-

\footnotetext{
${ }^{20}$ Como nos ha sugerido uno de los revisores de la revista, es necesario buscar argumentos independientes para reforzar la justificación de la existencia del SFu2 en las interrogativas atribuidas. A nuestro juicio, el que no sea en estos momentos suficientemente estándar la propuesta de un doble SFu se debe a que el carácter atribuido de la interrogativa tampoco ha sido abordado sintácticamente de modo regular. Aparte de que se apoya en la barrera prosódica especial que se observa ante el adverbio orientado al oyente en (18), la propuesta se enmarca en la investigación reciente que asume un doble SC en relación con ciertos fenómenos vinculados al dominio periférico oracional, p. ej., Escandell Vidal 2002 sobre las interrogativas de eco; Zanuttini y Portner 2003 sobre las exclamativas; Haegeman 2003a, 2006 sobre las condicionales; Demonte y Fernández Soriano 2004 sobre las construcciones de evidencialidad. El SFu2 puede considerarse como la proyección que encabeza los estratos equivalentes al SC inferior (cf. Rizzi 1997).
} 
das (cf. §1.2). En este subapartado intentaremos un acercamiento más detallado a esta cuestión. Para comodidad del lector, reproducimos de nuevo los ejemplos de (2) como (22):

(22) a. *Juan afirma que, francamente, le da miedo la oscuridad.

b. *Juan pregunta a Ana si, sinceramente, le da miedo la oscuridad.

La formulación en estilo indirecto de una oración con AAH desencadena agramaticalidad. Imaginemos que una persona llamada Juan está profiriendo la oración (23a). Si se formula (23a) en estilo indirecto, como hacemos en (23b), el resultado es agramatical pese a observarse una simple reproducción fiel de la otra oración:

(23) a. Sinceramente, René va a ser el mejor Wotan de la época.

b. *Juan afirma que, sinceramente, René va a ser el mejor Wotan de la época.

La única manera de mantener el anclaje de la oración original en el hablante consistiría en representarla como cita en estilo directo, como se utiliza con frecuencia en la conversación espontánea:

(24) Juan dice: «Sinceramente, René va a ser el mejor Wotan de la época».

Estos ejemplos sugieren que los AAHs solo pueden legitimarse en la posición periférica de la cláusula matriz. Se trata de un fenómeno bien extraño, si tenemos en cuenta que se asume normalmente en la gramática que las cláusulas matrices y las subordinadas disponen de una misma periferia izquierda. A este respecto, proponemos que, al contrario de la idea generalizada, hay ciertas diferencias composicionales de gran relevancia en la organización de la periferia izquierda de estos dos tipos de cláusulas, dependiendo de su capacidad o no para contener fuerza ilocutiva. La legitimación de los AAHs depende, entonces, de la codificación de dicha fuerza en la proyección de la periferia izquierda.

\subsection{La disociación de la fuerza ilocutiva y el sistema del complementante}

El Sintagma Fuerza se denomina así porque codifica la información que especifica la fuerza ilocutiva de la oración (Rizzi 1997). Todas las cláusulas matrices expresan cierta fuerza ilocutiva (Searle 1969) y, por tanto, es razonable asumir que el SFu las encabeza. Sin embargo, normalmente se acepta que esta proyección acoge igualmente a los complementantes subordinantes. Es bien sabido, por otro lado, que las cláusulas subordinadas no poseen fuerza ilocutiva, limitándose a 
denotar el estado de $\operatorname{cosas}^{21}$. Como consecuencia no deberíamos cometer el error de considerar idénticos los dos tipos de cláusulas en lo que respecta a la capacidad de codificar la fuerza ilocutiva. Veamos la configuración de una oración imperativa representada de manera convencional:

(25) [SFu [ST confiese usted [SFu que [ST nos ha mentido]]]]

Si la fuerza directiva está codificada en la periferia de la cláusula matriz, resulta cuestionable identificar y etiquetar la proyección que encabeza a la cláusula subordinada como SFu. De esta observación surge una inmediata necesidad de subclasificar la periferia izquierda.

La fuerza ilocutiva se define como la intención del hablante de producir el enunciado (Holdcroft 1978) y, por tanto, es el origen que desencadena las construcciones lingüísticas. Se trata de un constituyente de nivel abstracto y sería difícil reducirla a determinados tipos de oraciones o modos. Sin embargo, se suele afirmar en la bibliografía que la morfología de C, p. ej., la de las declarativas, interrogativas, relativas, etc., manifiesta la fuerza de la oración (cf. Rizzi 1997, Chomsky 2000); de ahí la identificación entre fuerza ilocutiva y sistema C. El sistema de $\mathrm{C}$ de algunas lenguas en las que se distinguen tipos de oraciones o modos como el del saramaccano se menciona como prueba de esta hipótesis en muchas ocasiones (Aboh 2006, p. 32):

(26) a. Amato sábi $\{t a ́ a / *$ fu\} Ajawa kísi dí ógófou a mátu. Amato sabe que Ajawa captura DET buho LOC jungla 'Amato sabe que Ajawa capturó al buho en la jungla'.

b. Amato kě $\{f u / *$ táa $\}$ Ajawa kísi dí ógífu a mátu. Amato quiere que Ajawa captura DET buho LOC jungla 'Amato quiere que Ajawa capture al buho en la jungla'.

A nuestro juicio esta idea no encaja con el hecho de que las cláusulas subordinadas a las que pertenecen los complementantes subordinantes no tienen fuerza ilocutiva. Suponemos que la morfología de C

${ }^{21}$ Fraser 1974, p. 2, se opone a esta idea e insiste en que la fuerza ilocutiva se deja percibir idénticamente en las cláusulas subordinadas:

(i) a. I regret that I must inform you of your dismissal. 'Siento tener que informarte de tu despido'.

b. I am pleased to be able to offer you the job. 'Me alegro de poder ofrecerte el empleo'.

No obstante, la interpretación ilocutiva en estos ejemplos (informar sobre el despido, ofrecer el empleo) se obtiene por inferencia. Las cláusulas subordinadas solo manifiestan el estado de cosas del que el hablante se siente apenado y satisfecho, respectivamente. 
mantiene cierta relación selectiva con la fuerza ilocutiva de la cláusula matriz, pero no la codifica en $\mathbf{s i}^{22}$.

El supuesto de que los tipos de oraciones coinciden con la fuerza ilocutiva se revela igualmente en el hecho de que las partículas de algunas lenguas que manifiestan la distinción de tipos de oración se consideren como codificadores de fuerza. P. ej., ta y $n i$ del coreano indican que las oraciones que terminan con estas son declarativas e interrogativas, respectivamente ${ }^{23}$ :

(27) a. Taeyeon-un nolay-lul cal han-ta. Taeyeon-NOM canto-ACUS bien hacer-DECL 'Taeyeon canta bien'.

b. Taeyeon-un nolay-lul cal ha-ni? Taeyeon-NOM canto-ACUS bien hacer-INTERR ‘Canta bien Taeyeon?'

Se suele señalar que estas partículas ocupan su posición en el dominio periférico de la oración, de ahí que puedan contener la fuerza ilocutiva (cf. Haegeman 2006). Sin embargo, son capaces de aparecer libremente dentro de las cláusulas subordinadas con el mismo valor, como se ilustra en (28). Por tanto, ponemos en tela de juicio la idea de que las partículas llevan consigo la fuerza. Además, según Cinque 1999, es probable que sean constituyentes que pertenecen al dominio oracional sin necesidad de mirar hacia la periferia izquierda.

(28) a. Taeyeon-un nolay-lul cal han-ta-ko malhay-ss-ta. Taeyeon-NOM canto-ACUS bien hacer-DECL-que decir-PAS-DECL 'Han dicho que Taeyeon canta bien'.

b. Taeyeon-un nolay-lul cal ha-ni-a-ko mul-ess-ta. Taeyeon-NOM canto-ACUS bien hacer-INTERR-A-que preguntar-PAS-DECL 'Han preguntado si Taeyeon canta bien'.

La conclusión que puede extraerse de estos datos es la disociación inevitable de la fuerza ilocutiva y el sistema C. En otras palabras, abandonamos el supuesto de que los dos elementos se representan sintácticamente en una misma categoría. Proponemos que el SFu codifica

${ }^{22}$ Es interesante observar que en español el complementante subordinante que debe preceder ambas completivas coordinadas si no hay coincidencia de modos en estas -cf. (ii)-, lo que a su vez tendría que ver con distintas fuerzas de las cláusulas matrices -no tratamos en este trabajo cómo se expresa la fuerza que rige la segunda completiva en (ii)-:

(i) a. Mi hermana dice que va a preparar un plato especial y (que) le falta el aceite.

b. Mi hermana dice que vaya al supermercado y (que) le compre una botella de aceite.

(ii) Mi hermana dice que le falta el aceite * $^{*}$ que) le compre una botella de aceite.

${ }^{23}$ Utilizamos The Yale Romanization para la transcripción del coreano (cf. Martin 1992). 
únicamente la fuerza ilocutiva y que, por tanto, preside las cláusulas matrices. La configuración de la periferia izquierda de estas se resume tal y como hemos observado en las secciones anteriores:

$$
\text { [SFu }\left[\mathrm { SF } \left[\mathrm { SX } \left[\mathrm { SAH } \left[\mathrm{SAH}^{*}[\mathrm{STOp}[\mathrm{SFoc}[\mathrm{SFin} \ldots\right.\right.\right.\right.
$$

En cuanto a los complementantes, les asignamos una categoría exclusiva, que denominamos c para evitar posibles confusiones con el C convencional. El Sc, carente de cualquier fuerza ilocutiva, es incapaz de seleccionar a $\mathrm{SAH}$, que es la proyección relacionada con el acto de habla. La periferia izquierda de las cláusulas subordinadas se estructuraría del siguiente modo ${ }^{24}$ :

(30) $[$ Sc $[$ STop $[$ SFoc $[$ SFin ...

Llegados a este punto, parece conveniente llamar la atención sobre el asunto que hemos dejado pendiente antes, es decir, la jerarquía entre SFu y SAH (cf. §2.2). Hemos ido argumentando a favor de que el SFu es la proyección jerárquicamente más alta en la periferia izquierda en contra de Hill 2007, quien considera que el SAH y SAH* son superiores al SFu. El análisis de la autora se fundamenta en el comportamiento del marcador del discurso hai. De origen turco, está muy extendido en lenguas de la península balcánica y algunas eslavas, y denota el valor exhortativo o evaluativo/evidencial -cf. (31)-. Hill se concentra en su variación en rumano, que considera una materialización del núcleo $\mathrm{AH}^{*}$, y que resulta inaceptable en las oraciones subordinadas, como se muestra en (32) (Hill 2007, p. 2094) ${ }^{25}$ :

(31) Hai cã m-a zãpăcit.

HAI que me ha confundido

'Vamos, porque él/ella me ha confundido. /

Definitivamente, él/ella me ha confundido'.

(32) *Maria spune cã hai cã Ion nu mai vine.

Maria dice que HAI que Ion no más viene.

${ }^{24}$ Haegeman 2002, 2003a, 2003b, 2006, distingue también dos periferias izquierdas diferentes para las oraciones subordinadas adverbiales según la codificación de la fuerza izquierda. Nuestra proyección funcional Sc se correspondería con el Sintagma Subordinante de la autora. Recordamos al lector que la configuración completa de las cláusulas subordinadas requiere un estudio más detallado. Se ha propuesto en la bibliografía que el margen izquierdo de estas cláusulas puede tener una estructura todavía más empobrecida que la que se presenta en (30) (cf. Haegeman 2003a, Hernanz 2006). Sin pretender ser exhaustivos sobre la representación concreta de la periferia izquierda de las cláusulas subordinadas, nos limitaremos a hacer hincapié en la idea de que esta carece de las categorías funcionales más altas que inciden en el acto de habla.

${ }_{25}$ Véase Hill 2007, pp. 2091-2098, para la justificación de hai como núcleo AH*. 
$\mathrm{Al}$ asumir el enfoque tradicional de que los complementantes ocupan el núcleo del SFu, Hill se ve obligada a concluir que el SAH* queda fuera de esta proyección. Sin embargo, esto ya no será necesario si enfocamos la cuestión valiéndonos de una periferia izquierda revisada como la nuestra, que permite mantener la propuesta, avalada empírica y conceptualmente, de que SFu es la proyección más alta de la periferia izquierda. La agramaticalidad de (32) se debe a la periferia izquierda truncada de las cláusulas subordinadas, que carecen de un $\mathrm{SAH}^{*}$ que aloje el marcador hai. Completamos de este modo nuestra propuesta sobre la ordenación del dominio de la interficie sintaxis-discurso.

\subsection{Prohibición de los adverbios de actos de habla y otras predicciones}

El hecho de que las cláusulas subordinadas no toleren a los AAHs se explica, entonces, de manera semejante a como se ilegitima hai en estas cláusulas. La presencia y la orientación de estos adverbios requieren ciertas categorías funcionales en la periferia izquierda -cf. (29)-. Las cláusulas subordinadas, que no cuentan con esas categorías, no pueden admitirlos ${ }^{26}$.

Por otra parte, nuestra propuesta predice acertadamente que la presencia de los vocativos y exclamativos en las cláusulas subordinadas provoca agramaticalidad, porque necesitan también las proyecciones incorporadas en la periferia izquierda con la fuerza ilocutiva como SAH y $\mathrm{SAH}^{*}$ (cf. §2.2):

(33) a. *Las noticias afirman que, María, va a llover esta tarde.

b. * YYa te he advertido varias veces de que, hijo, no hagas eso!

c. *¿Puedes preguntarle si, majo, me puede prestar aquel libro?

(34) a. *El taxista murmuró que, por Dios, no conocía esa calle.

b. *Avisan que, madre mía, las entradas ya están agotadas.

c. *¿Te parecerá normal que, anda, ese niño se queje de todo?

${ }^{26}$ Aunque difiere de nuestro estudio en el detalle, también Haegeman 2010 señala que la existencia de la fuerza ilocutiva en la cláusula es un requisito para la admisión de los AAHs. Por otra parte, un revisor anónimo nos hace la interesante observación de que tal vez algunos verbos como confesar o manifestar puedan permitir que los AAHs en la cláusula subordinada se orienten al sujeto de la cláusula matriz, sobre todo, cuando se realizan en pasado:

(i) a. ?Juan confesó que, francamente, no le apetecía nada venir a la fiesta.

b. ?Juan manifestó que, sinceramente, la elección no le parecería adecuada.

Este hecho sugiere que el tiempo verbal parece jugar un papel relevante en ciertos casos y que, probablemente, cada clase de verbo proyecte una estructura funcional diferente. Hay que señalar que estos juicios contrastan con los de nuestros informantes, que motivaron lo expuesto en la nota 5 , pero indudablemente nos obligan a hacer un examen más a fondo y más sutil para todas las variedades posibles del español. Abordaremos esta añadida complejidad empírica en una futura investigación. 
Por último, la propuesta presentada en este trabajo caracteriza adecuadamente los cambios que tienen lugar con la conversión a estilo indirecto abordada en el subapartado 5.1. Este proceso no solo consiste en la modificación de las expresiones referenciales, sino también en la pérdida de la fuerza ilocutiva que tenía el enunciado en cuanto cláusula matriz. Se demuestra así que la distinción entre la periferia izquierda de la cláusula matriz y la de la cláusula subordinada se ajusta a los datos examinados, y que debe abandonarse una concepción unificada para ambas.

\section{Conclusiones}

En este trabajo nos hemos propuesto un estudio formal de la orientación y distribución de los adverbios de actos de habla. Con una nueva perspectiva, hemos tratado de ampliar y refinar la periferia izquierda como interficie sintaxis-discurso, en la cual la fuerza ilocutiva sintácticamente codificada y una serie de proyecciones funcionales inciden directamente en la interpretación y distribución de estos adverbios, que son una manifestación léxica de los estratos en cuestión. De este modo hemos podido observar también que idéntica computación a la observable en la Sintaxis Estricta rige para la interficie sintaxis-discurso y que los fenómenos examinados pueden ser explicados adecuadamente sin necesidad de estipular ninguna condición adicional.

\section{REFERENCIAS BIBLIOGRÁFICAS}

Авон, E. (2006): «Complementation in Saramaccan and Gungbe: The case of C-type modal particles», Natural Language and Linguistic Theory 24, pp. 1-55.

Alexiadou, A.; Anagnostopoulou, E. y SCHÄFer, F. (2006): «The properties of anticausatives crosslinguistically», en Frascarelli, M. (ed.), Phrases of interpretation, Berlín, Mouton de Gruyter, pp. 175-199.

ANDERSON, S. R. (1971): On the linguistic status of the performative/constative distinction, Bloomington, Indiana University Linguistics Club Publication.

BÁez SAN José, V. (1994): «Las formas en -mente en una teoría fenomenológica integrada del acto de hablar, la expresión y el esquema oracional», Lingüística Española Actual 16, pp. 229-269.

BELLERT, I. (1977): «On the semantic and distributional properties of sentential adverbs», Linguistic Inquiry 8, pp. 337-351.

Chomsky, N. (1995): The minimalist program, Cambridge (Mass.), MIT Press.

- (2000): «Minimalist inquiries: The framework», en Matin, R. Michaels, D. y Uriagereka, J. (eds.), Step by step: Essays on minimalist syntax in honor of Howard Lasnik, Cambridge (Mass.), MIT Press, pp. 89-153.

- (2001): «Derivation by phase», en Kenstowicz, M. (ed.), Ken Hale: A life in language, Cambridge (Mass.), MIT Press, pp. 1-52. 
CINQUe, G. (1999): Adverbs and functional heads. A cross-linguistic perspective, Nueva York, Oxford University Press.

- (2004): «Issues in adverbial syntax», Lingua 114, pp. 683-710.

Demonte, V. y Fernández Soriano, O. (2004): «Features in comp and syntactic variation: the case of '(de)queísmo' in Spanish», Lingua 115, pp. 1063-1082.

Dik, S.; Hengeveld, K.; Vester E. y Vet, C. (1990): «The hierachical structure of the clause and the typology of adverbial satellites», en Jan Nuyts, A.; Bolkestein, M. y Vet, C. (eds.), Layers and levels of representation in language theory, Ámsterdam, John Benjamins, pp. 25-70.

ERnst, T. (2002): The syntax of adjuncts, Cambridge, Cambridge University Press.

ESCANDELL VIDAL, V. (1999): «Los enunciados interrogativos. Aspectos semánticos y pragmáticos», en Bosque, I. y Demonte, V. (eds.), Gramática descriptiva de la lengua española, Madrid, RAE-Espasa-Calpe, pp. 3929-3992.

- (2002): «Echo-syntax and metarepresentations», Lingua 12, pp. 871-900.

Fraser, B. (1974): «An examination of the performative analysis», Papers in Linguistics 7, pp. 1-40.

HaEgeman, L. (2002): «Anchoring to speaker, adverbial clauses and the structure of CP», en Mauck, S. y Mittelstaedt, J. (eds.), Georgetown University Working Papers in Theoretical Linguistics 2, pp. 117-180.

- (2003a): «Conditional clauses: External and internal syntax», Mind \& Language 18, pp. 317-339.

- (2003b): «Speculations on adverbial fronting and the left periphery», en Guéron, J. y Tasmowski, L. (eds.), Temps et point de vue/Tense and point of view, París, Publidix, pp. 329-365.

- (2006): «Conditionals, factives and the left periphery», Lingua 116, pp. 1651-1669.

- (2010): «The movement derivation of conditional clauses», Linguistic Inquiry 41 , pp. 595-621.

Halle, M. (1997): «Distributed morphology: Impoverishment and fission», en Bruening, B.; Kang, Y. y Mcginnis, M. (eds.), MITWPL 30: Papers at the interface, pp. 425-449.

HeNGEVELD, K. (1990): «The hierarchical structure of utterance», en Jan Nuyts, A.; Bolkestein, M. y Vet, C. (eds.), Layers and levels of representation in language theory, Ámsterdam, John Benjamins, pp. 1-23.

Hernanz, M. L. (2006): «Emphatic polarity and $C$ in Spanish», en Brugè, L. (ed.), Studies in Spanish syntax, Venecia, Università Ca' Foscari, pp. 105-150.

Hill, V. (2007): «Vocatives and the pragmatics-syntax interface», Lingua 117, pp. 2077-2105.

Holdcroft, D. (1978): Words and deeds: Problems in the theory of speech acts, Nueva York, Oxford University Press.

Hornstein, N. (2009): A theory of syntax, Cambridge, Cambridge University Press.

JACKENDOFF, R. (1972): Semantic interpretation in generative grammar, Cambridge (Mass.), MIT Press.

KALULLI, D. (2007): «Rethinking the passive/anticausative distinction», Linguistic Inquiry 38, pp. 770-780. 
Kaul de Marlangeon, S. B. (2002): Los adverbios en -mente del español de hoy y su función semántica de cuantificación, Madrid, Iberamericana.

KAYNE, R. S. (1994): The antisymmetry of syntax, Cambridge (Mass.), MIT Press.

Kovacci, O. (1991): El comentario gramatical, vol. 2, Madrid, Arco/Libros.

- (1999): «El adverbio», en Bosque, I. y Demonte, V. (eds.), Gramática descriptiva de la lengua española, Madrid, RAE-Espasa-Calpe, pp. 740-778.

LARSON, R. (1988): «On the double object construction», Linguistic Inquiry 19, pp. 335-391.

LONGOBARDI, G. (1994): «Reference and proper names: A theory of N-movement in syntax and logical form», Linguistic Inquiry 25, pp. 609-665.

Martin, S. E. (1992): A Korean reference grammar, Rutland, VT/Tokyo, Charles E. Tuttle.

Nissenbaum, J. (2000): Investigations of covert phrase movement, tesis doctoral, MIT.

Pesetsky, D. y Torrego, E. (2007): «The syntax of valuation and the interpretability of features», en Karimi, S.; Samiian, V. y Wilkins, W. (eds.), Phrasal and clausal architecture, Ámsterdam, John Benjamins, pp. 262-294.

Rizzi, L. (1997): «The fine structure of left periphery», en Haegeman, L. (ed.), Elements of grammar, Dordrecht, Kluwer, pp. 281-337.

Rodríguez RAmalle, T. M. (2003): La gramática de los adverbios en -mente o cómo expresar maneras, opiniones y actitudes a través de la lengua, Madrid, Universidad Autónoma de Madrid.

RoEHRs, D. (2006): The morpho-syntax of the germanic noun phrase: Determiners move into the determiner phrase, tesis doctoral, Indiana University.

Ross, J. R. (1970): «On declarative sentences», en Roderick, J. y Rosenbaum, R. (eds.), Readings in English transformational grammar, Waltham, Ginn, pp. 222272.

SADOCK, J. M. (1974): Towards a linguistic theory of speech acts, Nueva York, Academic Press.

SEARLE, J. R. (1969): Speech acts, Cambridge, Cambridge University Press.

SELLS, P. (1987): «Aspects of logophoricity», Linguistic Inquiry 18, pp. 445-479.

SPEAS, M. (2004): «Evidentiality, logophoricity and the syntactic representation of pragmatic features», Lingua 114, pp. 255-276.

- y TenNy, C. (2003): «Configurational properties of point of view roles», en Di Sciullo, A. M. (ed.), Asymmetry in grammar; syntax and semantics, Ámsterdam, John Benjamins, pp. 315-344.

TORner Castells, S. (2005): Aspectos de la semántica de los adverbios de modo en español, tesis doctoral, Universitat Pompeu Fabra.

Torrego, E. y UriagereKa, J. (1992): Indicative dependents, manuscrito, University of Massachusetts Boston y University of Maryland.

ZANutTini, R. y PORTner, P. (2003): «Exclamative clauses: At the syntax-semantics interface», Language 79, pp. 39-79. 
(05) - Art. Yoon Kim. RSEL 42-2: (7) -Revista Ac. nㅇł 14/1/13 12:24 Página 152 